\title{
Orthorhombic rational approximants for decagonal quasicrystals
}

\author{
S RANGANATHAN and ANANDH SUBRAMANIAM* \\ Department of Metallurgy, Indian Institute of Science, Bangalore 560 012, India \\ *Regional Research Laboratory, Bhopal 462 026, India
}

MS received 22 April 2003; revised 24 June 2003

\begin{abstract}
An important exercise in the study of rational approximants is to derive their metric, especially in relation to the corresponding quasicrystal or the underlying clusters. Kuo's model has been the widely accepted model to calculate the metric of the decagonal approximants. Using an alternate model, the metric of the approximants and other complex structures with the icosahedral cluster are explained elsewhere. In this work a comparison is made between the two models bringing out their equivalence. Further, using the concept of average lattices, a modified model is proposed.
\end{abstract}

Keywords. Decagonal quasicrystals; orthorhombic rational approximants; average lattices.

\section{Introduction}

The discovery of quasicrystals (Shechtman et al 1984) marked such a major paradigm shift in the understanding of structure of ordered matter that time old tenets of crystallography had to be drastically expanded. Soon after the discovery, the early descriptions of quasicrystalline diffraction patterns were based on three models: (i) quasiperiodic tilings (Levine and Steinhardt 1984), (ii) icosahedral glasses (Stephens 1989) and (iii) twinning effects (Pauling 1985). The icosahedral glass models were a compromise between the observed icosahedral symmetry and the impossibility to tile three-dimensional space with icosahedra, while the ability of a twinned aggregate to mimic a system of higher symmetry was the basis for the third kind of model. Currently cluster based models are gaining currency, wherein the role played by atoms initially is played by a cluster of atoms, which are hierarchically arranged (Janot 1997). However, it is important to take note of the recent discovery of Abe et al (1999), where they report a decagonal quasicrystal in the $\mathrm{Mg}$ Zn-Dy system without atomic clusters.

The next two major breakthroughs in the field came with the discovery of lower dimensional quasicrystals (Chattopadhyay et al 1985) and the realization of some well known complex crystals as rational approximants to quasicrystals (Elser and Henley 1985). Rational approximants coexist with quasicrystals in most samples and often it is difficult to distinguish higher order approximants from quasicrystals. When approximants form by the transformation from quasicrystals, the aggregate retains the symmetry of the parent quasicrystal. Hence an invol-

*Author for correspondence ved analysis is required to distinguish the cases of quasicrystals, rational approximants and twinned aggregates. Ranganathan et al (1997) have written a comprehensive review on decagonal quasicrystals and their rational approximants.

An important exercise in the study of rational approximants is to derive their metric, especially in relation to the corresponding quasicrystal or the underlying clusters. Two approaches can be used: (i) projection formalism, wherein a hyperdimensional cube is projected along a rational slope (Henley 1985; Mandal and Lele 1994) and (ii) working in physical space and using the icosahedral clusters (Shoemaker and Shoemaker 1988). The latter approach has an intuitive appeal in that it allows the visualization of icosahedral linkages.

The importance of the icosahedral cluster and its linkages in various complex crystalline structures finally leading to the quasicrystal have been studied in detail before. Shoemaker and Shoemaker (1988) described the various polyhedra with icosahedral symmetry and their occurrence in alloy phases like $\mathrm{NaZn}_{13}, \mathrm{Mg}_{2} \mathrm{Zn}_{11}, \mathrm{MoAl}_{12}$ etc. In the present context their description of permeation of icosahedral order through vertex, edge and face sharing and the different orientations of the icosahedron generated in the process are of relevance. They have stated: 'we may suspect that the sizes of the unit cells are determined in many cases, at least in part, by requirements imposed by the linking of icosahedra'.

Using the concept of 'canonical cells' Henley (1991) attempted to construct the structure of the icosahedral phase. Focusing on clusters connected by linkages and understanding the local environments, he has derived various geometrical properties of the lattice like packing fraction and coordination number and has tried to describe the structure of rational approximants. His model is 
a nice merger between tiling based and cluster based models and the attempt to derive the lattice parameters of the crystal from the dimensions of the basic cluster is noteworthy.

Zhang and Kuo (1990a) proposed a model for obtaining the rational approximants to the decagonal quasicrystal by the projection formalism. This has been the widely accepted model so far. Anantharaman (1999) proposed an alternate model to explain approximants and other complex structures with the icosahedral cluster. In this work a comparison is made between the two models bringing out their equivalence. Further, a modified model is proposed.

\section{Kuo's model}

Orthorhombic approximants have been discovered for icosahedral, decagonal and hexagonal quasicrystals. Kuo using linear phason strain theory first studied the transformation of decagonal quasicrystal to an one-dimensional quasicrystal (Zhang and Kuo 1990a) and then proceeded to derive the lattice parameters for the orthorhombic approximants to the decagonal phase within the same framework (Zhang and Kuo 1990b). The theory attempts to account for the observed lattice parameters for cubic, orthorhombic and monoclinic approximants.

The lattice parameters for the orthorhombic approximants to the decagonal phase are given by (Zhang and Kuo 1990b):

$$
\begin{aligned}
& a=a_{\mathrm{D}}=\sqrt{ } 5 a_{\mathrm{R}} \tau^{n+1} /\left(1+\tau^{2}\right)^{1 / 2}, \\
& c=a_{\mathrm{P}}=\sqrt{ } 5 a_{\mathrm{R}} \tau^{n},
\end{aligned}
$$

where, $a_{\mathrm{R}}$ is the edge length of the Penrose rhombus, $a_{\mathrm{D}}$ the lattice parameter along the original true two-fold of the decagonal quasicrystal, $a_{\mathrm{P}}$ the lattice parameter along the direction orthogonal to $a_{\mathrm{D}}$ and $\tau$ the golden mean $(=(1+\sqrt{ } 5) / 2)$.

Based on equal or unequal order of approximations along ' $a$ ' and ' $c$ ' the orthorhombic approximants to the decagonal phase can be classified into two types: the Taylor and Robinson approximants. As seen from (1), there is a $\tau$ inflation of lattice parameters from one order of approximant to another (table 1). The common periodicities observed along the ' $b$ ' axis for the decagonal quasicrystal are 1, 2, 3 and 4. A rare periodicity of 9 is also observed (Okabe et al 1992). The lattice parameter along this periodic axis is inherited by the corresponding orthorhombic rational approximant.

\section{Anantharaman's model}

Anantharaman (1999) used the icosahedral cluster as the basic building unit. Starting with an orthorhombic cell, which essentially contains an icosahedron, various structures (crystalline, approximant and quasicrystalline) are generated by putting together an integral number of basic cells. These vertex, edge and face sharing icosahedra are assumed to suffer distortions to give rise to cubic, tetragonal, hexagonal, rhombohedral or monoclinic structures. Interplanar spacing data from Debye-Scherrer diffraction experiments of $\mathrm{Al}-\mathrm{Cu}-\mathrm{Fe}$ icosahedral quasicrystal is compared with the model.

\section{Comparison between Anantharaman's and Kuo's models}

In the current analysis the orthorhombic approximants to the decagonal quasicrystal are considered and a comparison is made between Anantharaman's and Kuo's (Zhang and Kuo 1990a) models. Further, a modified model based on Anantharaman's approach is proposed, which can explain the discrepancies arising out of Anantharaman's model and bring it closer to Kuo's model.

For a detailed analysis of Anantharaman's model we start with the table 4 in his paper (Anantharaman 1999). The table differentiates between three classes of cluster compounds adding up to thirty-six: crystalline, quasicrystalline (icosahedral and decagonal) and rational approximants. For consistency with that accepted in literature, further discussion is restricted to the twenty-eight phases listed under crystalline (item numbers 1-10) and rational approximants (item numbers 18-35). The phases identified as crystalline by Anantharaman are in fact rational approximants to the decagonal phase with the exception of $\varepsilon-\mathrm{Al}_{4} \mathrm{Cr}$ which is a hexagonal approximant. For a fruitful comparison between Anantharaman's and Kuo's models we set aside the $\varepsilon-\mathrm{Al}_{4} \mathrm{Cr}$ phase. For comparison of Kuo's values of lattice parameters for the decagonal approximants with that of Anantharaman's, the ' $a$ ' and ' $c$ ' in Anantharaman's table have been re-listed taking into account the orientation of the true two-fold axis in the corresponding decagonal phase (table 2). The cases of switch between $a_{\mathrm{D}}$ and $a_{\mathrm{P}}$ (rows 5-7 in Anantharaman's table) are also now in good match with integral multipliers (table 3). Notwithstanding certain anomalies it can be seen that the various ' $m$ ' and ' $n$ ' (the modified values are listed as $m^{\prime}$ and $n^{\prime}$ ) values can be correlated with various orders of approximants under Kuo's scheme.

Table 1. Lattice parameters derived from Kuo's model (Zhang and Kuo 1990b).

\begin{tabular}{lcrr}
\hline$N$ & $F_{m} / F_{m-1}$ & $a_{\mathrm{D}}$ & \multicolumn{1}{c}{$a_{\mathrm{P}}$} \\
\hline 1 & $1 / 0$ & $0 \cdot 76$ & $0 \cdot 90$ \\
2 & $1 / 1$ & 1.23 & $1 \cdot 45$ \\
3 & $2 / 1$ & 1.99 & $2 \cdot 34$ \\
4 & $3 / 2$ & $3 \cdot 23$ & 3.79 \\
5 & $5 / 3$ & $5 \cdot 22$ & $6 \cdot 13$ \\
6 & $8 / 5$ & 8.44 & 9.92 \\
7 & $13 / 8$ & 13.65 & $16 \cdot 04$ \\
8 & $21 / 13$ & 22.09 & 25.96 \\
\hline
\end{tabular}


Table 2. Correlation between order of approximant (Kuo's) and Anantharaman's 'm' and 'o' values.

\begin{tabular}{|c|c|c|c|c|c|c|c|c|c|c|c|}
\hline & & & & $\begin{array}{c}a(\mathrm{~nm}) \\
\left(a_{\mathrm{D}}\right)\end{array}$ & $\begin{array}{c}b \\
(\mathrm{~nm})\end{array}$ & $\begin{array}{c}c(\mathrm{~nm}) \\
\left(a_{\mathrm{P}}\right)\end{array}$ & $(d, p)$ & $o^{\prime}$ & $m^{\prime}$ & $x$ & $y$ \\
\hline 1 & Robinson (1952) & $\mathrm{R}$ & $\mathrm{Al}_{21} \mathrm{Mn}_{3} \mathrm{Cu}_{2}$ & $0 \cdot 772$ & $1 \cdot 250$ & $2 \cdot 42$ & $(1 / 0,2 / 1)$ & 3 & 6 & 3 & 8 \\
\hline 2 & Robinson (1954) & $\mathrm{R}$ & $\mathrm{Al}_{31} \mathrm{Mn}_{6} \mathrm{Ni}_{2}$ & $0 \cdot 755$ & $1 \cdot 250$ & $2 \cdot 380$ & $(1 / 0,2 / 1)$ & 3 & 6 & 3 & 8 \\
\hline 3 & Damjanovic (1961) & $\mathrm{R}$ & $\mathrm{Al}_{27} \mathrm{Mn}_{7} \mathrm{Zn}_{5}$ & $0 \cdot 778$ & $1 \cdot 260$ & $2 \cdot 380$ & $(1 / 0,2 / 1)$ & 3 & 6 & 3 & 8 \\
\hline 4 & Ellner (1995) & $\mathrm{R}$ & $\mathrm{Al}_{10} \mathrm{Fe}_{3}$ & 0.775 & $0 \cdot 403$ & $2 \cdot 377$ & $(1 / 0,2 / 1)$ & 3 & 6 & 3 & 8 \\
\hline 5 & Taylor $(1960)^{*}$ & $\mathrm{~T}$ & $\mathrm{Al}_{13} \mathrm{Mn}_{4}\left(\mathrm{Al}_{10} \mathrm{Mn}_{3}\right)$ & $1 \cdot 259$ & $1 \cdot 242$ & $1 \cdot 48$ & $(1 / 1,1 / 1)$ & $3 * *$ & $6^{* *}$ & 5 & 5 \\
\hline 6 & Burkhardt et al (1996)* & $\mathrm{R}$ & $\mathrm{Al}_{13} \mathrm{Co}_{4}\left(\mathrm{Al}_{10} \mathrm{Co}_{3}\right)$ & $0 \cdot 816$ & $1 \cdot 234$ & $1 \cdot 445$ & $(1 / 0,1 / 1)$ & $2 * *$ & $6^{* *}$ & $3 * *$ & 5 \\
\hline 7 & Hiraga et al (1993)* & $\mathrm{T}$ & $\mathrm{Al}_{3} \mathrm{Mn}\left(\mathrm{Al}_{28} \mathrm{Mn}_{11}\right)$ & $1 \cdot 243$ & $1 \cdot 251$ & $1 \cdot 483$ & $(1 / 1,1 / 1)$ & $3 * *$ & $6 * *$ & 5 & 5 \\
\hline 8 & Li et al (1994) & $\mathrm{R}$ & $\mathrm{Al}_{3} \mathrm{Co}\left(\mathrm{Al}_{28} \mathrm{Co}_{11}\right)$ & $1 \cdot 250$ & $1 \cdot 250$ & $0 \cdot 810$ & $(1 / 1,1 / 0)$ & 5 & 2 & 5 & 3 \\
\hline 9 & Ma et al (1990) & $\mathrm{R}$ & $\mathrm{Al}_{3} \mathrm{Pd}$ & $1 \cdot 230$ & $1 \cdot 670$ & $2 \cdot 340$ & $(1 / 1,2 / 1)$ & 5 & 6 & 5 & 8 \\
\hline 18 & Li and Kuo (1992a) & $\mathrm{R}$ & $\Pi-\mathrm{Al}_{4} \mathrm{Mn}$ & $0 \cdot 770$ & $1 \cdot 26$ & $2 \cdot 360$ & $(1 / 0,2 / 1)$ & 3 & 6 & 3 & 8 \\
\hline 19 & Van Tendeloo et al (1988) & $\mathrm{R}$ & $\mathrm{C}_{3 \mathrm{I}}-\mathrm{Al}_{60} \mathrm{Mn}_{11} \mathrm{Ni}_{4}$ & $3 \cdot 270$ & $1 \cdot 240$ & $2 \cdot 400$ & $(3 / 2,2 / 1)$ & 13 & 6 & 13 & 8 \\
\hline 20 & Zhang et al (1995) & $\mathrm{R}$ & $\mathrm{Al}_{75} \mathrm{Pd}_{13} \mathrm{Ru}_{12}$ & $3 \cdot 280$ & 1.669 & $2 \cdot 388$ & $(3 / 2,2 / 1)$ & 13 & 6 & 13 & 8 \\
\hline 21 & Yu et al (1993) & $\mathrm{R}$ & $\mathrm{C}-1 \mathrm{Al}_{70} \mathrm{Co}_{15} \mathrm{Ni}_{10} \mathrm{~Tb}_{5}$ & $5 \cdot 460$ & $1 \cdot 600$ & $2 \cdot 280$ & $(5 / 3,2 / 1)$ & $21^{* *}$ & $6^{* *}$ & $21 * *$ & 8 \\
\hline 22 & Yu et al (1993) & $\mathrm{R}$ & $\mathrm{C}-2 \mathrm{Al}_{70} \mathrm{Co}_{15} \mathrm{Ni}_{10} \mathrm{~Tb}_{5}$ & $8 \cdot 400$ & $0 \cdot 400$ & $6 \cdot 100$ & $(8 / 5,5 / 3)$ & 34 & $16^{* *}$ & $34 * *$ & 21 \\
\hline 23 & Yu et al (1994) & $\mathrm{T}$ & C-3 $\mathrm{Al}_{70} \mathrm{Co}_{15} \mathrm{Ni}_{10} \mathrm{~Tb}_{5}$ & $3 \cdot 200$ & $1 \cdot 600$ & $3 \cdot 680$ & $(3 / 2,3 / 2)$ & 13 & 9 & 13 & 13 \\
\hline 24 & Yu et al (1994) & $\mathrm{T}$ & C-4 $\mathrm{Al}_{70} \mathrm{Co}_{15} \mathrm{Ni}_{10} \mathrm{~Tb}_{5}$ & $2 \cdot 000$ & 1.600 & $2 \cdot 360$ & $(2 / 1,2 / 1)$ & 8 & 6 & 8 & 8 \\
\hline 25 & Yu et al (1994) & $\mathrm{R}$ & C- $5 \mathrm{Al}_{70} \mathrm{Co}_{15} \mathrm{Ni}_{10} \mathrm{~Tb}_{5}$ & $1 \cdot 260$ & 1.600 & $2 \cdot 300$ & $(1 / 1,2 / 1)$ & 5 & 6 & 3 & 8 \\
\hline 26 & Okabe et al (1992) & $\mathrm{R}$ & $\mathrm{I}-\mathrm{Al}_{70} \mathrm{Cr}_{20} \mathrm{Cu}_{10}$ & $3 \cdot 300$ & $3 \cdot 780$ & $2 \cdot 400$ & $(3 / 2,2 / 1)$ & 13 & 6 & 13 & 8 \\
\hline 27 & Okabe et al (1992) & $\mathrm{R}$ & II- $\mathrm{Al}_{70} \mathrm{Cr}_{20} \mathrm{Cu}_{10}$ & $2 \cdot 040$ & $3 \cdot 780$ & $9 \cdot 420$ & $(2 / 1,8 / 5)$ & 8 & $24 * *$ & 8 & $34 * *$ \\
\hline 28 & Wu et al (1996) & $\mathrm{R}$ & $\mathrm{I}-\mathrm{Al}_{67} \mathrm{Cu}_{18} \mathrm{Cr}_{25}$ & $0 \cdot 780$ & $1 \cdot 240$ & $2 \cdot 370$ & $(1 / 0,2 / 1)$ & 3 & 6 & 3 & 8 \\
\hline 29 & Wu et al (1996) & $\mathrm{R}$ & II- $\mathrm{Al}_{67} \mathrm{Cu}_{18} \mathrm{Cr}_{25}$ & 1.970 & $3 \cdot 720$ & $6 \cdot 140$ & $(2 / 1,5 / 3)$ & 8 & $16^{* *}$ & 8 & 21 \\
\hline 30 & Liao et al (1992) & $\mathrm{T}$ & $\mathrm{I}-\mathrm{Al}_{65} \mathrm{Cu}_{20} \mathrm{Co}_{15}$ & 1.970 & $0 \cdot 400$ & $2 \cdot 330$ & $(2 / 1,2 / 1)$ & 8 & 6 & 8 & 8 \\
\hline 31 & Liao et al (1992) & $\mathrm{R}$ & II- $\mathrm{Al}_{65} \mathrm{Cu}_{20} \mathrm{Co}_{15}$ & $5 \cdot 200$ & $0 \cdot 410$ & $3 \cdot 800$ & $(5 / 3,3 / 2)$ & 21 & $10 * *$ & 21 & 13 \\
\hline 32 & Launois et al (1990) & $\mathrm{R}$ & III- $\mathrm{Al}_{65} \mathrm{Cu}_{20} \mathrm{Fe}_{15}$ (I) & $3 \cdot 188$ & 0.827 & $9 \cdot 811$ & $(3 / 2,8 / 5)$ & $13^{* *}$ & $24 * *$ & $13^{* * *}$ & 34 \\
\hline 33 & Launois et al (1990) & $\mathrm{R}$ & IV- $\mathrm{Al}_{65} \mathrm{Cu}_{20} \mathrm{Co}_{15}$ & $8 \cdot 440$ & 0.410 & $6 \cdot 140$ & $(8 / 5,5 / 3)$ & 34 & $16^{* *}$ & 34 & 21 \\
\hline 34 & Dong et al (1992) & $\mathrm{R}$ & $\mathrm{Al}_{65} \mathrm{Cu}_{20} \mathrm{Fe}_{15}(\mathrm{I})$ & $3 \cdot 250$ & $1 \cdot 228$ & $2 \cdot 365$ & $(3 / 2,2 / 1)$ & 13 & 6 & 13 & 8 \\
\hline 35 & Dong et al (1992) & $\mathrm{R}$ & $\mathrm{Al}_{65} \mathrm{Cu}_{20} \mathrm{Fe}_{10} \mathrm{Cr}_{5}$ & $2 \cdot 010$ & $1 \cdot 227$ & $6 \cdot 194$ & $(2 / 1,5 / 3)$ & 8 & $16^{* *}$ & 8 & 21 \\
\hline 37 & $\mathrm{Li}$ and Kuo (1992b) & $\mathrm{T}$ & $\mathrm{Y}-\mathrm{AlMnCu}$ & $1 \cdot 26$ & $1 \cdot 24$ & 1.48 & $(1 / 1,1 / 1)$ & 5 & $6^{* *}$ & 5 & 5 \\
\hline 38 & Li et al (1992) & $\mathrm{T}$ & $\mathrm{Y}-\mathrm{Al}_{3} \mathrm{Mn}$ & $1 \cdot 256$ & $1 \cdot 247$ & 1.487 & $(1 / 1,1 / 1)$ & 5 & $6^{* *}$ & 5 & 5 \\
\hline 39 & Hiraga et al (1993) & $\mathrm{T}$ & $\mathrm{Al}_{70} \mathrm{Pd}_{5} \mathrm{Mn}_{25}$ & $1 \cdot 251$ & $1 \cdot 243$ & 1.483 & $(1 / 1,1 / 1)$ & 5 & $6 * *$ & 5 & 5 \\
\hline 40 & Van Tendeloo et al (1988) & $\mathrm{R}$ & $\mathrm{C}_{3, \mathrm{II}}-\mathrm{AlMnNi}$ & $1 \cdot 26$ & $1 \cdot 24$ & $2 \cdot 68$ & $(1 / 1,2 / 1)$ & 5 & $7 * *$ & 5 & $8 * *$ \\
\hline
\end{tabular}

Key: numbers in the first column correspond to item numbers in table 4 from Anantharaman (1999); *the ' $a$ ' and ' $c$ ' values are such that $a=a_{\mathrm{D}}$ and $c=a_{\mathrm{P}} ; *$ not exact match.

Table 3. Recalculation of the multiplying factors based on the revised list.

\begin{tabular}{|c|c|c|c|c|c|c|c|c|c|c|}
\hline & Reference & Alloy & $a$ & $b$ & $c$ & $(d, p)$ & $m$ & $o$ & $m^{\prime}$ & $o^{\prime}$ \\
\hline 5 & Taylor (1960) & $\mathrm{Al}_{13} \mathrm{Mn}_{4}\left(\mathrm{Al}_{10} \mathrm{Mn}_{3}\right)$ & $1 \cdot 242^{+}$ & $1 \cdot 259^{+}$ & $1 \cdot 479$ & $(1 / 1,1 / 1)$ & 3 & 6 & 5 & 5 \\
\hline 6 & Burkhardt et al (1996) & $\mathrm{Al}_{13} \mathrm{Co}_{4}\left(\mathrm{Al}_{10} \mathrm{Co}_{3}\right)$ & $0 \cdot 816$ & $1 \cdot 234$ & $1 \cdot 445$ & $(1 / 0,1 / 1)$ & 2 & 6 & 3 & 5 \\
\hline 7 & Hiraga et al (1993) & $\mathrm{Al}_{3} \mathrm{Mn}\left(\mathrm{Al}_{28} \mathrm{Mn}_{11}\right)$ & $1 \cdot 243$ & $1 \cdot 251$ & 1.483 & $(1 / 1,1 / 1)$ & 3 & 6 & 5 & 5 \\
\hline
\end{tabular}

In the model of Anantharaman, wherein periodic stacking of the basic unit cell has been adopted, the increased order of the approximant is reflected as Fibonacci sequence of ' $m$ ' and ' $n$ ' values. The paper fails to address the reason behind these special values taken by the multiplying factors. This can be seen by the close correspondence of the lattice parameters of these phases with that derived by Kuo's formula. In this work a $\tau$ deflation has been used on the values derived by (1) to include phases with smaller lattice parameters in the analysis.

\section{The average lattice}

Even after the advent of quasicrystals, recourse is often taken to the intuitively appealing concept of a periodic lattice in diverse ways. The advantage that the well developed tools of crystallography can be put to use is an added benefit. Modulation of a basic lattice, displacively or occupationally, can be used to generate a variety of structures. Modulation functions incommensurate with the basic lattice gives rise to aperiodic structures. Godreche 
and Oguey (1990) used the projection method to obtain average lattices for quasiperiodic structures. Baranidharan (1997) has systematically introduced the concept of the average lattice and has suggested a method of applying the same to the icosahedral quasicrystal.

It can be shown that (table 4) the occurrence of Fibonacci numbers for multiplying factors and the postulation of basic lattice parameter are closely linked. If successive $\tau$ inflated values of a unit lattice parameter are divided by the corresponding Fibonacci number (of cells) the resulting sequence converges to a constant value (akin to the ratio of successive terms in a standard Fibonacci sequence):

$$
\lim _{n \rightarrow \infty} \frac{\tau^{n}}{F_{n+2}}=\lim _{n \rightarrow \infty} \frac{F_{n} \tau+F_{n-1}}{F_{n+2}}=\frac{1}{\tau}+\frac{1}{\tau^{3}}=0 \cdot 854102 \ldots
$$

This value to which the ratio converges can be numerically used as a fundamental unit and integral multiples of this unit approximates the approximant parameter better with increasing order of the approximant. Various $\tau$ inflated or deflated values of the one derived in (1) can be considered as a lattice parameter as well. Wolny (1998) considered an average cell parameter of $1 \cdot 382\left(=1+1 / \tau^{2}\right)$ in his study of diffraction properties of one-dimensional average lattices.

Steurer and Haibach (1999) considered the construction of average lattices in detail and have derived the average lattice parameter for $1 D, 2 D$ and $3 D$ quasiperiodic lattices. For the Penrose tiling they have derived

$$
a_{1}^{\mathrm{avg}}=(3-\tau) a_{\mathrm{r}} \quad a_{2}^{\mathrm{avg}}=(3-\tau)^{3 / 2} a_{\mathrm{r}} / \tau,
$$

where, $a_{1}^{\text {avg }}$ is parallel to $a_{\mathrm{D}}$ and $a_{2}^{\text {avg }}$ is parallel to $a_{\mathrm{P}}$. Let

$$
(3-\tau)=\left(1+1 / \tau^{2}\right)=x_{\mathrm{avg}}=1 \cdot 3819,
$$

with, $\sqrt{ } x_{\text {avg }}=1 \cdot 1755 \ldots$ (and $\left.\sin ^{-1}\left(\sqrt{ } x_{\text {avg }} / 2\right)=36^{\circ}\right)$ being the smaller diagonal of the fat rhombus in the Penrose tiling. This length scale is obtained by a section along $\mathbf{a}_{1}$

Table 4. Derivation of average lattice parameter based on $\tau$ inflation of the lattice parameter and the Fibonacci sequence.

\begin{tabular}{lccc}
\hline $\begin{array}{l}\text { Order of } \\
\text { rational } \\
\text { approximant }(n)\end{array}$ & $\begin{array}{c}\text { Repeating } \\
\text { units }\left(F_{n}\right)\end{array}$ & $\begin{array}{c}\text { RAs to } \\
\text { quit cell }\left(\tau^{n-1}\right)\end{array}$ & $\begin{array}{c}\text { Average lattice } \\
\text { unantharaman }) \\
\left(\tau^{n-1} / F_{n}\right)\end{array}$ \\
\hline 1 & & 1 & 1 \\
2 & 1 & $\tau=1 \cdot 618$ & $1 \cdot 618 .$. \\
3 & 2 & $\tau^{2}=2 \cdot 618$ & $1 \cdot 309 .$. \\
4 & 3 & $\tau^{3}=4 \cdot 236$ & $1 \cdot 412 .$. \\
5 & 5 & $\tau^{4}=6 \cdot 854$ & $1 \cdot 370 .$. \\
6 & 8 & $\tau^{5}=11 \cdot 09$ & $1 \cdot 386 .$. \\
7 & 13 & $\tau^{6}=17.94$ & $1 \cdot 380 .$. \\
8 & 21 & $\tau^{7}=29.03$ & $1 \cdot 382 .$. \\
$n \rightarrow \infty$ & $\infty$ & $\infty$ & $1+1 / \tau^{2}$ \\
\hline
\end{tabular}

in the Penrose tiling and forms a small unit in the Fibonacci sequence.

Comparing with Kuo's model

$$
\frac{a_{\mathrm{P}}}{a_{\mathrm{D}}}=\frac{\sqrt{1+\tau^{2}}}{\tau}=1 \cdot 1755 \ldots
$$

and

$$
\frac{a_{2}^{\mathrm{avg}}}{a_{1}^{\mathrm{avg}}}=\frac{\sqrt{3-\tau}}{\tau}=\frac{1 \cdot 1755 \ldots}{\tau} .
$$

Also defining

$$
a_{\mathrm{D}}^{\prime}=\frac{a_{\mathrm{D}}}{\tau^{n}} \quad \text { and } \quad a_{\mathrm{P}}^{\prime}=\frac{a_{\mathrm{P}}}{\tau^{n}}
$$

Steurer's parameters can be compared with those of Kuo

$$
\frac{a_{1}^{\mathrm{avg}}}{a_{\mathrm{D}}^{\prime}}=\frac{1 \cdot 1755 \ldots}{\tau} \text { and } \quad \frac{a_{2}^{\mathrm{avg}}}{a_{\mathrm{P}}^{\prime}}=\frac{1 \cdot 1755 \ldots}{\tau^{2}} .
$$

Hence the $\tau$ factor deflation is seen along $\mathbf{a}_{1}$ in Steurer's model as compared to Kuo's model.

Anantharaman though implicitly has used the concept of an average lattice parameter, but, he has failed to take into account the occurrence of two different units in the Fibonacci sequence in the actual structure.

\section{A modified model}

The idea of Anantharaman can be modified by starting with a different set of lattice parameters as in table 5 . Taking into account the additional $\tau$ factor deflation along $\mathbf{a}_{1}$ these new values are derived from Steurer's values as follows

$$
a_{\mathrm{mod}}=a_{1}^{\mathrm{avg}} / \tau x_{\mathrm{avg}} \approx 0.25 \text { and } c_{\mathrm{mod}}=c_{1}^{\mathrm{avg}} / x_{\mathrm{avg}} \approx 0.29 .
$$

Also, the new set of values are integrally related to Kuo's values and the multiplying factors derived by their use are listed in table 2. The anomalous occurrence of nonFibonacci numbers for the multipliers in Anantharaman's model are now accounted for and the idea of an average basic cell can be understood within the framework of quasicrystallography.

Table 5. Comparison of orthorhombic unit cell dimensions from Kuo's model (Wu et al 1996), Anantharaman's model (1999) and the modified model.

\begin{tabular}{lccc}
\hline Kuo's model & $\begin{array}{c}\text { Steurer's model } \\
\text { (Penrose tiling) }\end{array}$ & $\begin{array}{c}\text { Anantharaman's } \\
\text { model }\end{array}$ & $\begin{array}{c}\text { Modified } \\
\text { model }\end{array}$ \\
\hline$a=a_{\mathrm{D}}{ }^{\prime}=0.76$ & $a_{1}{ }^{\text {avg }}=0.55$ & $c_{\mathrm{TRA}}=0.25$ & $a_{\text {mod }}=0.25$ \\
$b=b=0.4$ & - & $b_{\mathrm{TRA}}=0.419$ & $b_{\text {mod }}=0.4$ \\
$c=a_{\mathrm{P}}{ }^{\prime}=0.90$ & $a_{2}{ }^{\text {avg }}=0.40$ & $a_{\mathrm{TRA}}=0.40$ & $c_{\text {mod }}=0.29$ \\
\hline
\end{tabular}




\section{Conclusions}

Anantharaman has restated Kuo's ideas by invoking an integrally assembled orthorhombic unit cell in the Fibonacci sequence. As seen in table 4 with increasing order of the approximant the 'average lattice parameter' of the fundamental cell converges, thus forming an apparently convincing argument towards a fundamental cell and masking the occurrence of $\tau$. A modified model based on Anantharaman's idea can be envisaged which can better explain the lattice parameter data for orthorhombic approximants. Finally, the most important aspect is that the actual structural arrangement follows the Fibonacci sequence within the unit cell in a recursive and nonrepetitive mode.

\section{Acknowledgement}

The authors would like to thank Prof. T R Anantharaman for his thought-provoking and sometimes provocative discussions.

\section{References}

Abe E, Sato T J and Tsai A P 1999 Phys. Rev. Lett. 825269 Anantharaman T R 1999 Bull. Mater. Sci. 22937

Baranidharan S 1997 Prog. Cryst. Growth \& Charact. 34157

Burkhardt U, Ellner M and Grin Y 1996 Powder Diffraction 11123

Chattopadhyay K, Lele S, Ranganathan S, Subbanna G N and Thangaraj N 1985 Curr. Sci. 54895

Damjanovic A 1961 Acta Crystallogr. 14982

Dong C, Dubois J M, Kang S S and Audier M 1992 Philos. Mag. B65 107

Ellner M 1995 Acta Crystallogr. B51 31

Elser V and Henley C L 1985 Phys. Rev. Lett. 552883

Godreche C and Oguey C J 1990 Phys. France 5121

Henley C L 1985 J. Non-Crystallogr. Solids 7591

Henley C L 1991 Phys. Rev. B43 993

Hiraga K, Kaneko M, Matsuo Y and Hashimoto S 1993 Philos. Mag. B67 193
Janot C 1997 J. Phys. Condens. Matter 91493

Launois P, Audier M, Denoyer F, Dong C, Dubois J M and Lambert M 1990 Europhys. Lett. 13629

Levine D and Steinhardt P J 1984 Phys. Rev. Lett. 532477

Liao X Z, Kuo K H, Zhang H and Urban K 1992 Philos. Mag. B66 549

Li X Z and Kuo K H 1992a Philos. Mag. B65 525

Li X Z and Kuo K H 1992b Philos. Mag. B66 117

Li X Z, Shi D and Kuo K H 1992 Philos. Mag. B70 331

Li X Z, Ma X L and Kuo K H 1994 Philos. Mag. Lett. 70221

Ma L N, Wang R and Kuo K H 1990 J. Less-Common Metals 16337

Mandal R K and Lele S 1994 Proc. int. conf. aperiodic crystals (eds) G Chapuis and W Paciorek (Singapore: World Scientific)

Okabe T, Furihata J I, Morishita K and Fujimori H 1992 Philos. Mag. Lett. 66259

Pauling L 1985 Nature 317512

Ranganathan S, Chattopadhyay K, Singh A and Kelton K F 1997 Prog. Mater. Sci. 41195

Robinson K 1952 Philos. Mag. 43775

Robinson K 1954 Acta Crystallogr. B7 494

Shechtman D, Blech I, Gratias D and Cahn J W 1984 Phys. Rev. Lett. $\mathbf{5 3} 1951$

Shoemaker D P and Shoemaker C B 1988 Introduction to quasicrystals (ed.) M V Jaric (Boston: Academic Press Inc.) p. 1

Stephens P 1989 Extended icosahedral structures (eds) M V Jaric and D Gratias (San Diego: Academic Press) p. 37

Steurer W and Haibach T 1999 Acta Crystallogr. A55 48

Taylor M A 1960 Acta Metall. 8256

Van Tendeloo G, Van Landuyt J, Amelinkx S and Ranganathan S 1988 J. Microsc. 1491

Wen K Y, Chen Y L and Kuo K H 1992 Met. Trans. A23 2437

Wolny J 1998 Acta Crystallogr. A54 1014

Wu J S, Ma X L and Kuo K H 1996 Philos. Mag. Lett. 73 163

Yu R C, Li X Z, Xu D P, Zhang Z, Su W H and Kuo K H 1993 Philos. Mag. Lett. 67287

Yu R C, Li X Z, Xu D P, Zhang Z, Su W H and Kuo K H 1994 Scr. Metall. Mater. 311285

Zhang H and Kuo K H 1990a Phys. Rev. B41 3482

Zhang H and Kuo K H 1990b Phys. Rev. B42 8907

Zhang B, Li X Z, Steurer W, Schneider J and Frey F 1995 Philos. Mag. Lett. 72239 